\title{
Accessing Antenatal Care (ANC) services during the COVID-19 First Wave - Insights into decision- making process from rural India
}

Shweta Bankar ( $\sim$ shwetabankar@gmail.com )

Ashoka University https://orcid.org/0000-0003-2219-980X

Deepika Ghosh

Ashoka University

\section{Research Article}

Keywords: Antenatal Care Services, Behavioral principles, Childbirth, COVID-19, India, Pregnant Women

Posted Date: January 31st, 2022

DOI: https://doi.org/10.21203/rs.3.rs-1273382/v1

License: (c) (1) This work is licensed under a Creative Commons Attribution 4.0 International License.

Read Full License 
4 Corresponding and First Author: Shweta Bankar [SB], Senior Research Fellow, Centre for Social and Behaviour Change, Ashoka University, Haryana, India

6

7 Second Author: Deepika Ghosh [DG], Deputy Programme Manager, Centre for Social and 8 Behaviour Change, Ashoka University, Haryana, India 
Abstract

27

28

29

30

31

32

\section{Background}

Antenatal Care (ANC) services are a prime focus of the National Health Mission (NHM) of the Government of India, with emphasis on promoting maternal and child health. The services are designed to reach villages with the appointment of Frontline Health Workers (FLHWs) and the establishment of a network of health centres at the primary level for uninterrupted service delivery.

The decision to access these services was highly impacted by the onset COVID-19 pandemic and the outcomes of the national lockdown from late March to June 2020.

\section{Methods}

Using a qualitative design, data were collected through 12 in-depth interviews (IDIs) with pregnant women and 17 IDIs with frontline health workers in the selected six districts under study. The narratives were analysed using inductive coding in Atlas.ti.

\section{Results}

Most of whom belonged to poor and marginalised groups, and pregnant women were left with limited access to health centres and FLHWs. Respondents from the study areas of rural Jharkhand, Madhya Pradesh and Uttar Pradesh extensively reported concerns stemming from the lockdown that influenced their decision around accessing ANC services. These included anxieties around meeting their families' daily needs due to a loss of livelihood (in particular, abject food insecurity), inability to access healthcare, and a sense of mistrust in public health systems and functionaries. 
45 These, coupled with the real threat to health posed by COVID-19, threw their plans for pregnancy

46 and delivery into jeopardy.

\section{Conclusion}

48 This study identified a number of behavioural, social and structural factors deeply entrenched in the

49 communities, which resulted in confusion and anxiety, leaving pregnant women and FLHWs

50 feeling hapless and helpless. To ensure ANC services in times of crisis, strategies to address the

51 behavioural, social and structural concerns and place ANC services as essential services in the study

52 area and in similar contexts need to consider the factors identified by this study.

\section{Key Words}

54 Antenatal Care Services, Behavioral principles, Childbirth, COVID-19, India, Pregnant Women

\section{Plain English Summary}

56 This article highlights the status of antenatal care services during the countywide lockdown in the

57 first COVID-19 wave in March 2020 in Jharkhand, Madhya Pradesh and Uttar Pradesh. Data were

collected through 12 in-depth interviews with pregnant women and 17 in-depth interviews with

59 frontline health workers. Findings depict pregnant women were aware of the severity of the COVID-

6019 situation and the protective measures through their trusted sources of information, especially the

61 ASHAs. However, both pregnant women and frontline health workers considered the information wasn't sufficient, and there were a lot of unanswered questions, especially about their pregnancy and

63 childbirth. Temporary suspension of the health services resulted in confusion and distress, leaving pregnant women unaware of the health status of the fetus. Limited or no interaction with the 
65

frontline health workers with reports of lack of attention towards the non-COVID-19 illnesses/conditions and discrimination at healthcare institutions increased the uncertainties towards institutional/safe deliveries in the public health care. This was further out byzantine by the loss of livelihood given the pandemic and the lockdown as the respondents and their families could not count the option of childbirth in private hospitals resulting in deterioration trust in the public health care system. To bridge the gap and decrease the distrust the pandemic has created in its endusers, strategies to improve the utilisation of health services and address other issues identified in this article should be considered.

\section{Background}

Maternal mortality is a priority area under the United Nations' Sustainable Development Goal (SDG) 3, aiming to ensure the health and promote well-being for people of all ages (1). The Government of India is committed to lowering the Maternal Mortality Ratio (MMR) from 130 (maternal deaths per 1,00,000 live births) in 2016 to 70 by 2030 (2). Considering the extensive challenges in the provision of the comprehensive approach to reduction of MMR, ANC services and the access to safe deliveries, especially in rural areas, were strengthened. The Government of India (GoI) has designed and implemented interventions to improve the reach and quality of ANC services and augment demand for these services. Programs implemented in the last 15 years include the National Rural Health Mission (2005) (3), the National Urban Health Mission (2008) (4), and the Reproductive, Maternal, New-born, Child, and Adolescent Health (RMNCH+A) Strategy, introduced in 2013 (5). There has been a marked improvement in the uptake of these services in the 
last ten years, given the government's efforts and sustained engagement of frontline health workers (6-8). Most recently, in 2016-18, India's MMR was down to 113 per 1,00,000 live births, yet there is a long way to go (9). The percentage of pregnant women attending at least four ANC contacts increased from $43.9 \%$ (NFHS-3) to 58.6\% (NFHS-4) (10). As per NFHS-5 data released for 22 Indian states and UTs, the percentage of women availing of ANC services in the first trimester has increased in 18 states/ UTs, and the percenage of women completing the recommended minimum of four ANC visits has increased in 11 states/ UTs in the years since NFHS-4 (11).

In India, official guidance for pregnant women recommends a minimum of four antenatal care contacts during pregnancy, at specified intervals. However, during the COVID-19 pandemic, pregnant women were advised not to visit sites of ANC check-ups in April and May 2020. The Indian Council for Medical Research (ICMR) and National Institute for Research in Reproductive Health (NIRRH) issued a communication in the initial days of the lockdown that detailed the updated protocol for childbirth, such as reservation of obstetrics units for confirmed COVID-19 positive cases; and ensuring that the pregnant woman, Healthcare Workers (HCWs) and birth partner all wear PPE during labour and delivery (12). However, there was a gap in frontline health workers' awareness and comprehension of these guidelines, which led to an atmosphere of confusion and fear at the grassroots (13). An overall lack of preparedness in the form of PPE kits and infrastructure, administrative apathy, persistent socio-economic inequalities and resulting discrimination, and apathy towards returning migrants were barriers to accessing ANC services as the lockdown was eased $(13,14)$.

Besides the unavailability of check-up facilities, other services, chiefly home visits to pregnant women and distribution of Take-Home Ration (THR), also proved inaccessible. Supply-side 
barriers to access included disruption in the transportation of supplements like iron and folic acid (IFA), zinc and calcium tablets, the burden on health workers of COVID-19-related tasks, which reduced their availability for maternal healthcare service delivery (15). On the demand side, key barriers included inaccurate or incomplete information about service provision, fear of contracting COVID-19 due to stepping out of the home, widespread loss of livelihoods, restrictions on mobility, etc. $(16,17)$. Services were restored by $68-90 \%$ of FLHWs in July 2020 , and among them, immunisation was the first to resume, followed by home visits by the FLHW and distribution of supplementary nutrition (18).

Regional studies described a high awareness among pregnant women around precautionary behaviours to be adopted during COVID-19 (19,20). However, studies also brought to light pregnant women's anxieties around securing food and shelter in the absence of paid work, the suffering of their children due to school closure and confinement in the home, and lack of social support (among those belonging to ethnic minorities and other marginalised groups) (21). Even during the unlocking process, uptake of most ANC services did not improve due to constraints on travelling (such as the limited functioning of public transport, apprehension around using public transport due to chances of contracting infection, and lack of access to private vehicles for transportation to sites of ANC check-ups); non-availability of services; a general fear of contracting the disease when outdoors, which was heightened at the prospect of interface with healthcare functionaries and these influenced the decisions about the uptake of the services $(18,21)$

Lockdown in India aggravated women's perinatal anxiety, and they felt low confidence in giving birth during the pandemic. They were no longer able to avail of healthcare services as per the planned cadence of ANC check-ups and often had to forego attending them due to lack of reliable means of 
transportation, familial pressure to self-isolate, low-value perception of ANC in the eyes of key family members, fear of contracting the virus, vulnerability to complications in pregnancy, and risk of transmitting COVID-19 to the fetus, if they contracted the illness $(13,18)$. Due to the uncertainty around their families' wellbeing and the proliferation of unreliable sources of information, pregnant women were left anxious and impaired in their capacity to prepare for childbirth, which further exacerbated their distress (21).

Given the outcomes of the situation arising from the lockdown in India in the first COVID-19 phase, both at the systemic level and the personal level, we understand that the decisions around accessing non-COVID-19 health services were highly biased. This significantly impacted the access and utilisation of critical services like ANC and Safe Childbirth. This paper highlights the factors that influenced the uptake of ANC services and the institutional deliveries during the lockdown. We present our findings from a sample of 12 pregnant women and 18 Frontline Health Workers in rural India. The insights are drawn from the in-depth interviews conducted with the respondents.

\section{Methods}

This study is reported as per the Consolidated Criteria for Reporting Qualitative Research (COREQ) (22).

\section{Aim, design and setting of the study}

This qualitative research study was carried out by the Center for Social and Behavior Change (CSBC), Ashoka University, and funded by the Bill and Melinda Gates Foundation (BMGF). Jharkhand, Madhya Pradesh and Uttar Pradesh, India were selected as study locations due to 
151 CSBC's focus on these states as part of its ongoing partnership with the National Institute for

152 Transforming India (NITI) Aayog to improve health outcomes in these states. Two districts in each

153 of these states were selected for this study based on the presence of development partner

154 organisations in the districts. While preference was given to Aspirational Districts, the final selection

155 of districts was not limited to these.

156 This paper draws on data collected as part of a large-scale exploratory research study led by CSBC to

157 understand the landscape of health and nutrition services in COVID-19 in March 2020 and a phased

158 re-opening of the same in the selected states thereafter. The data was collected over three months to

159 grasp the status of services and the challenges faced by end-users and service providers during the

160 lockdown and Unlock 1.0. Ethical review and clearance were provided by the Institutional Review

161 Board of Ashoka University. A qualitative research design was adopted to study diverse aspects of

162 ANC services and institutional deliveries in these times.

163 This paper shares findings from 12 in-depth telephonic interviews conducted with pregnant women

164 and 17 in-depth interviews with frontline health workers. Pregnant women's interviews explored

165 themes of knowledge, awareness and practices in the times of COVID-19 pandemic; fears and

166 anxieties around pregnancy; the planning of childbirth and their needs for support (including from

167 frontline health workers); and the challenges anticipated during childbirth. In-depth telephonic

168 interviews with frontline health workers focussed on the change in their work schedule and

169 responsibilities owing to the pandemic, the institutional emphasis on preventing and managing

170 COVID-19, and its effect on the community, especially pregnant women. 
171 The interviews were conducted by the qualitative research team at CSBC (including the authors of 172 this paper) and trained field enumerators, and lasted approximately 45-60 minutes. Each of the 173 interview sessions was followed by a detailed debriefing. Interview guides were adapted to the 174 respondents' categories and comprised open-ended questions, followed by probes. At the same time, 175 some questions could be answered in the affirmative or negative, interviewers actively utilised probes to encourage further discussion around responses.

\section{Sample Characteristics}

178 Convenience sampling was undertaken of pregnant women while ensuring representation from three distinct sections of the community - general population, scheduled caste/tribes and returning migrants. Similarly, FLHWs were conveniently sampled, representing one Auxiliary Nurse Midwife (ANM), Accredited Social Health Activist (ASHA) worker, and Anganwadi worker (AWW) for each district. With the help of a matrix, each of the FLHWs was represented by one respondent from within the central district place and two from villages located far from the district centre. Table 1 outlines the inclusion criteria and distribution of the sample across the states. 
Table: 1 Inclusion Criteria and distribution of the sample

\begin{tabular}{|c|c|c|c|c|}
\hline $\begin{array}{l}\text { Respondent } \\
\text { Category }\end{array}$ & Inclusion Criteria & $\begin{array}{l}\text { Sample } \\
\text { Size }\end{array}$ & Distribution & $\begin{array}{l}\text { Number } \\
\text { of Units }\end{array}$ \\
\hline $\begin{array}{l}\text { Pregnant } \\
\text { Women }\end{array}$ & $\begin{array}{l}\text { Women in the second or third } \\
\text { trimester of pregnancy } \\
\text { accessing ANC services, } \\
\text { irrespective of the number of } \\
\text { previous pregnancies. }\end{array}$ & 12 & Pregnant woman per village & 12 villages \\
\hline $\begin{array}{l}\text { Frontline } \\
\text { Health } \\
\text { Workers }\end{array}$ & $\begin{array}{l}\text { Working FLHWs in each of } \\
\text { the selected districts }\end{array}$ & 17 & $\begin{array}{l}1 \text { Accredited Social Health } \\
\text { Activist (ASHA), } 1 \text { Auxiliary } \\
\text { Nurse Midwife (ANM) and } 1 \\
\text { Anganwadi Worker (AWW) } \\
\text { per district. }\end{array}$ & $\begin{array}{l}\text { Six } \\
\text { districts }\end{array}$ \\
\hline
\end{tabular}

Interviews were conducted in Hindi, Kho, and Bhojpuri at a time convenient for respondents and enumerators. Oral consent was obtained from each respondent before the interviews commenced. The enumerators took care to ensure that the telephonic engagement took place in a safe environment and maintained participants' confidentiality. All interviews were audiotaped, 
transcribed and translated verbatim into English by an external agency, and interviewers verified these translations to ensure accuracy.

\section{Data Analysis}

The thematic and narrative analyses (Braun and Clarke, 2006) were adopted to analyse the data collected. The team followed a step-wise process of familiarizing themselves with the data, identifying a thematic framework and developing a coding frame. In the preliminary stages, the data from notes taken by enumerators and CSBC researchers were thematically analyzed, to draw highlevel insights through a deductive approach. Further, the data was subjected to a thorough qualitative analysis with inductive coding, using Atlas.ti 9.0.

\section{Findings}

The findings highlight the different behavioural, financial, social and infrastructural factors that influenced pregnant women's and their families' decisions on the uptake of the ANC services and planning of safe deliveries.

\section{Pregnant Women's Knowledge and Risk Perception}

Pregnant women were aware of the COVID-19 situation in the country, its symptoms and the preventive measures. When asked if they knew of any other diseases that compared to COVID-19 in severity, the majority of respondents expressed that COVID-19 was the most formidable disease 
215

they had ever heard of due to its rapid spread, high rate of fatality, and that agencies had found no cure for the disease.

"There are other dangerous diseases, but there are solutions... there are medicines (for those diseases). But there is no treatment for this corona (sic), and that is why we are afraid." (Pregnant Woman,

$$
\text { District Khunti, Jharkhand) }
$$

A minority across the study areas said that this was just like the dozens of other dangerous diseases that were common, such as those borne by contaminated water. The high incidence of water-borne diseases in their community influenced their thinking. They feared that the concentration of efforts on the COVID-19 situation would delay timely and appropriate treatment of other illnesses and conditions, which may, in the absence of proper medical attention, prove fatal just like COVID-19.

There was a heightened awareness of COVID-19 being especially dangerous for pregnant women, as respondents had been told so by ASHAs. Some pregnant women across study areas reported receiving official text messages advising them to take special protective measures as they were especially vulnerable at this time. Respondents were emphatic that COVID-19 would adversely impact their health and the fetus' development but did not have any specific information on how this would happen. FLHWs confirmed this messaging and reported spreading these messages over phone or home visits.

$$
\begin{aligned}
& \text { "The ASHA told us ", If you stay at home, you will stay safe. Don't go to the hospital, (as) many } \\
& \text { different kinds of people are going there. It is unsafe for you, and for your child as well... that's } \\
& \text { why you should stay in your houses." (Pregnant Woman, District Lalitpur, Uttar Pradesh) }
\end{aligned}
$$


Pregnant women were aware of protective measures such as avoiding going out of their homes or consuming outside food, covering their faces with masks, washing hands with soap and water and maintaining social distance. FLHWs generated awareness about the COVID-19 situation and prevention along with routine pregnancy care guidance. During the lockdown, FLHWs typically communicated with them over phone calls and sometimes by visiting them at home. During home visits, FLHWs ensured no contact and followed all the other COVID-19 protocols. At the time of the interviews, in the early months of the COVID-19 lockdown in India, the effects of COVID-19 on pregnant women were unknown. Hence, there was no official guidance on the same as reported by an ASHA and reciprocated by other FLHWs across the study geographies.

"We have are to inform pregnant women about the COVID-19 situation and that they are bighly vulnerable to the infection, but we don't know how. Pregnant women ask us about this, and we have no answers.” (ASHA, District Chattarpur, Madhya Pradesh)

The respondents' other critical sources of information about the virus and pandemic included their mobile phones (via SMS, Government of India mandated COVID-19 advisory caller tune, and internet for those who owned smartphones), television and word of mouth.

\section{Anxieties around the pandemic}

Almost all pregnant women reported being acutely worried about making ends meet during the pandemic as this negatively impacted the nutrition of the family, and their special nutrition needs in their pregnancy. Owing to the lockdown, there was widespread loss of livelihoods, leading to abject insecurity around access to food. All respondents reported that their husbands were at home all day, 
without any work that would enable them to earn wages. With their children bound to the house, respondents felt that their education was suffering in the process. Concerns about their health during pregnancy, such as consuming a nutritious, well-balanced diet, were secondary to their families' daily anxieties about putting food on the table. This was especially true for respondents who did not have ration cards in the family and could not take advantage of the government's relief schemes. Even those who received rice under the government scheme (Pradhan Mantri Garib Kalyan Anna Yojana) said that it was grossly inadequate to meet the family's needs.

While some pregnant women received supplementary nutrition for their children, others whose children were over five years of age and no longer eligible for supplementary nutrition remarked that this further built-up pressure on them to feed their children. Since respondents were pregnant, they were not physically able to work and earn money for the household, which only compounded their stress. Some pregnant women were inconsolable as they narrated their hardships, having resorted to set of first-time expecting mothers reported feeling incredibly demoralised. They did not have relatives nearby to guide them and could not access ANC services (as well as guidance from health 
277

workers) during the lockdown. This contributed to a feeling of helplessness as these women faced dire material circumstances, including food insecurity, and were unable to remedy the situation.

\section{Access to Healthcare Services}

The suspended access to healthcare facilities, during the lockdown, and during the process of unlocking, pregnant women faced structural and behavioural challenges. ANMs recommend pregnant women to visit the Primary Health Centre (PHC) four times throughout their pregnancy. Pregnant women receive a host of services including physical examination, injections/immunization and supplements, investigations like blood tests and ultrasounds, as well as counselling on appropriate care at a Primary Health Centre (PHC), Anganwadi Centre (AWC) or monthly Village Health, Sanitation and Nutrition Day (VHSND). Respondents expressed their fears about availing these services at health centres even after taking due precautions since they could contract coronavirus from meeting others.

"I am afraid that it is possible for a pregnant woman to get corona easily. That's why we are... scared when we go to the hospital."(Pregnant Woman, District Khunti, Jharkhand)

Pregnant women were apprehensive of seeking healthcare services when it entailed travelling. Shutting down of public transport and usage of ambulances for transportation of COVID-19 positive persons increased their exposure to the virus. FLHWs further confirmed this as in the following quote,

\section{"We do not encourage pregnant women to use the ambulances which were actually for their use but} are now used to transport the COVID-19 patients due to shortage of vehicles. The ASHAs are 
In several cases, FLHWs discouraged the pregnant women from visiting health centres due to the

spread of COVID, instead recommended to seek phone consultation. In case ANC services were not being delivered at AWCs, pregnant women had no option but to turn to higher-level public health centres. Their fears around COVID-19 compounded with the belief that hospital staff would treat them poorly due to negative past experiences with institutional healthcare and rumours of discrimination and ill-treatment in the community.

Despite these apprehensions, there was angst at not accessing ANC services during the lockdown, as there was no way to know if the pregnancy was progressing normally and if the fetus was developing as it should.

"Doctors were saying that ultrasound is to be done twice... then only they come to know about the status of the baby and health of the mother. How can this be known if we don't consult the doctor?" felt it was essential their child's health. However, there was no mention of wanting to attend ANC contacts for the benefits to their health because, during pregnancy, the health of the baby is of utmost importance. In contrast, the health of the mother is essential to the extent that it impacts the baby's health, which leads to a devaluation of the latter. 
318 In some districts of Uttar Pradesh, pregnant women reported not being able to attend any ANC 319 check-ups due to the lockdown. doing checkups, so we came back...”(Pregnant Woman, District Lalitpur, Uttar Pradesh)

However, some pregnant women reported that ANC check-ups continued as usual, except that they received the vaccination, Mother and Child Protection (MCP) card and medicines (IFA, calcium, etc.) from a distance and the ANMs guided them to wear a mask or cover their face. Additionally, they were called into the AWC in small numbers for vaccination to prevent crowing. On rare occasions, pregnant women reported being given sanitiser to disinfect their hands prior to the meeting. vaccinations.”(Pregnant Woman, District Chitrakoot, Madhya Pradesh)

Of these women, some felt slighted by this new manner of service delivery, saying that they had to ask permission before entering the health centre, were told to sit at a distance from the ANM (which they felt was unnecessary), and were dealt with hurriedly. Some even said that while they were treated, as usual, the FLHWs' treatment of lower caste beneficiaries differed. 
In the first phase of unlocking (early May 2020), ANC services were being delivered by ANMs in their most rudimentary form at the AWC and prioritised immunisation and physical examination. IFA supplementation delivery varied from district to district. Some pregnant women reported receiving their share of IFA tablets from the AWC, others said that the AWC was shut. Hence, there was no supply of tablets, while still others said that they had not received any tablets from the AWC since the start of the pandemic but were yet to finish their stock from earlier. In many places, access to blood and urine tests, and weight checks was halted because of non-functioning infrastructure or because FLHWs did not have time to conduct them.

According to FLHWs, their engagement in the COVID-19 related tasks allowed very little time to cater to the ANC needs of pregnant women. They promoted tele-consulting as it saved time and effort and was the safest means in the given situation. With most of the services suspended, they could not cater to their ANC needs, and the supply disruption of the IFA, calcium and zinc tablets caused a shortage.

Several pregnant women in West Singhbhum (Jharkhand) and Chhatarpur (Uttar Pradesh) reported o being able to access ANC services and guidance at government hospitals often travelling long distances on a motorcycle in the heat and paying out of pocket for investigations like ultrasounds because the health functionaries were hesitant to enter the village due to the spread of 

and remind them to get vaccinated at the government hospital nearby but visited in person only once a month. This reminder was only issued for immunisation and not for any other services under the ANC umbrella after the unlocking process began towards early June in most of the districts under study.

In rare cases, during the lockdown, FLHWs guided and accompanied pregnant women to hospitals for health checks when the delivery of the services halted at AWCs. Others reported not being able to access any healthcare services in pregnancy during the lockdown as they had contacted their FLHW at the beginning of the lockdown, who informed them about the suspension of ANC services. Still, others mentioned that their frantic calls to their community's FLHWs, seeking clarity on the status of service delivery, had gone unanswered, which further contributed to a feeling of anxiety and hopelessness. in a burry, and finish off in a burry. We're not able to avail benefits of services like earlier."

Some women, who usually visited private hospitals, found that services like ultrasounds were not available in these facilities during the lockdown and hence were compelled to turn to government hospitals. These women usually belonged to a relatively higher socio-economic group (compared to the majority of respondents) and may have felt that services delivered were of higher quality in these private facilities. Pregnant women who felt forced to visit crowded and lower quality (in their perception) government hospitals during the pandemic, felt stressed. 
378 Respondents felt that the sporadic frequency of service delivery by FLHWs was not adequate for them. Not being able to meet FLHWs in person limited the usefulness of their interaction. This may 380 have been because those services that were perceived to be the most valuable (such as lab investigations like ultrasounds and blood tests) were not being administered. In the case of those who could only contact FLHWs over the phone, had no access to physical examination. Some pregnant women also reported not having adequate clarity on the timing of FLHWs' availability in the village and being confused on whom to approach for guidance in their absence, which led to additional confusion and a feeling of alienation from the health system. They reported seeking information on their mobiles (via internet search) or contacting the ASHA over the phone for recommendations of medicines and care practices. Others were discouraged and lost faith in FLHWs, turning to others for assistance.

A few pregnant women reported not utilising ANC services even before COVID-induced lockdown. They cited reasons such as not feeling they were valuable, not being permitted by their in-laws to attend, and so on), so they were not impacted in terms to a great degree by the lockdown.

Nearly all the respondents reported a strong preference for institutional deliveries, owing to the birth supervision by trained medical personnel and access to vital information on caring for their new- 
399

400

401

born. They mentioned that by giving birth in the hospital, they would receive necessary medication for themselves and the new-born. Some even said that delivering in a hospital brought the added advantage of helping them qualify for assistance under the government's maternal health schemes.

\section{"If deliveries happen at home, where will we get information from (about the schemes and} benefits)? When we go to the hospital, we will get information and services, and can take advantage of government schemes. (If we deliver at home), we won't get anything." (Pregnant Woman, District Lalitpur, Uttar Pradesh)

Some respondents discussed the experiences of women who had delivered children in their community during the pandemic. These new mothers had reportedly opted for institutional delivery and had not encountered any difficulties. Some differences were reported in the protocol followed at the onset of labour and during childbirth; only one family member was allowed to accompany the pregnant woman to the hospital, while there were no such restrictions before the pandemic. This resulted in anxieties for pregnant women as it was either the husband accompanying them in cases of lack of transport or ambulance to reach the hospitals without a female family companion. In contrast, they anticipated other women family member to accompany them whom they depicted signs of distrust in the absence of the husband.

When at the hospitals, women in labour were kept further apart than was usual and their discharge was at the earliest possible time after delivery. When pregnant women were apprehensive as they were unsure of receiving proper and sufficient treatment. Some FLHWs reported that COVID-19 testing was mandatory for pregnant women before admission in the hospital for delivery, but respondents could not corroborate this. 
When respondents (in particular, those who preferred institutional deliveries) spoke of their plans for delivery in the thick of the COVID-19 pandemic, they expressed trepidation since they were unsure of whether they would be able to give birth in the hospital and what the process would be. The uncertainty was because of the limited or no access to the regular course of institutional ANC services during their pregnancy and decreased FLHW interactions. may not happen, we don't know."(Pregnant Woman, District Chitrakoot, Uttar Pradesh) nervous about the process of childbirth because they did not know what steps to take if they started experiencing labour pains. This sentiment by even those pregnant women who had received checkups and ultrasounds and were nearing their expected delivery date. They claimed that since the lockdown, all pregnant women in their locality had delivered in hospitals, not at home. Despite their fear of contracting the virus and confusion about the process, they certainly preferred delivery in health facilities over home birth. onset of labour and how they planned to go to the hospital for delivery, given that they were apprehensive of using the ambulance. There was a deep sense of fear and confusion without any accompanying guidance on navigating the delivery. Due to the unprecedented and inexperienced 
conditions created by COVID-19 (such as constraints on mobility and limited access to healthcare functionaries and facilities), women could not look to their elders or community for guidance on managing their pregnancies. For these reasons, pregnant women felt demoralised and confused, without any clear course of action.

\section{Discussion}

The COVID-19 pandemic has irrevocably changed lives worldwide, and women's experience of pregnancy and childbirth has been no exception. Wide-ranging studies are presenting the impact of the COVID-19 pandemic on ANC service provision and access to services. Some of them bring to surface knowledge, risk perception, anxieties about service delivery, structural barriers aggravating the problem for pregnant women and FLHWs across the globe. In this setting, the behavioural barriers or enablers play an important role. In this piece, we discuss how underlying behavioural principles played a part in supporting the components in the findings of this article. Behavioural principles affected both service provision by the healthcare system and utilisation of services by the end-users at times aggravating barriers to access maternal health services and influencing the planning of safe deliveries. Factors such as knowledge about the pandemic, trust in the health system and availability of resources influenced the behaviour principles.

There was a mixed reaction to COVID-19 among pregnant women. In our study on the impact of the pandemic and consequent lockdown on the lives of families in Uttar Pradesh, Madhya Pradesh and Jharkhand, we observed an overwhelming sense of fear and confusion. Across study areas, pregnant women expressed apprehension about availing ANC services at health facilities due to fear of contracting COVID-19, as they believed it was dangerous for them and their unborn child. 
Though a small number of pregnant women did think of it to be not as dangerous as was purported, they did follow all the COVID-19 protocols to keep themselves away from the infection. All the participating pregnant women valued ANC visits. However, they supported the closure of the health care centres and restricted themselves to homes to be safe. In many cases, pregnant women felt hapless and feared the negative implications of lack of ANC services on the health and well-being of the fetus. Pregnant women felt incredibly vulnerable and anxious due to this half-knowledge, i.e., while they knew that COVID-19 posed a unique threat to them, they were left without any accompanying guidance on how to negotiate access to critical healthcare services during their pregnancy. This hampered pregnant women's physical and mental well-being with minimal scope for help and hope.

In the early months of the COVID-19 lockdown in India, the effects of the disease on pregnant women were unknown, and hence there was no official guidance. This lack of clarity may have heightened the anxieties of FLHWs, pregnant women and their families since pregnancy is a time of uncertainty and high stakes. But pregnant women and their families adhered to the instructions given their trust in mainly ASHAs. Whereas the FLHWs weren't comfortable spreading the information they had as they found themselves in situations that led to the loss of confidence when trying to answer queries from the pregnant women, especially around the effect of COVID-19 on the fetus. This led FLHW to avoid such situations resulting in decreased interaction with the endusers. Furthermore, this restricted FLHWs' decision-making ability and autonomy. This paved way to diminishing trust in the FLHWs by the end-users (despite their having known and trusted them for long), as the Pregnant Women sensed the discomfort within the FLHWs. This is an example of recency bias, wherein the end users favoured recent observations (i.e., FLHWs lacking 
conclusive information or guidance regarding COVID-19) over earlier observations (i.e., trust in

486 FLHWs, experienced healthcare providers for the community for a long time).

487 Pregnant women could see that the impact of COVID-19 was managed utilising the resources of the

488 public healthcare machinery. Hence, they could not see any positives resulting from this resource

489 reallocation. This is an example of the behavioural principle of loss aversion, where people

490 experience losses far more acutely than they experience gains, which was the fact of minimal

491 COVID-19 cases in the community due to quick deployment of frontline workers for community

awareness.

493 Respondents felt the pandemic, and the myriad of protective measures it brought, created conditions for authority figures (like ANMs) to actively act on their prejudices based on caste and classend-users in the guise of enforcing social distancing guidelines. While in some cases, caste-based discrimination may indeed have been meted out, in many cases, it was simply presumed that social distancing guidelines were being enforced in line with the practice of untouchability. This was an instance of confirmation bias on pregnant women, as they viewed real-life events (i.e. being asked to keep distance) as confirming pre-existing beliefs (i.e. the practice of untouchability).

As we understand from the findings, nearly all the pregnant women reported a loss of income in the family given the pandemic and the lockdown that followed to curb it. This was one of the reported stressors for the family, given that the special needs of the pregnant women and the plans around childbirth were disordered. Loss of income caused a lot more distress than the fear of COVID-19. This directly impacted fulfilling the special needs of pregnant women, medical aid and access to private health care. While in some districts, services like immunisation and basic physical examination were delivered in a most basic, hurried manner, in others, there was no response from 

and district government hospitals depending on their financial resources, while others did not receive any antenatal care. Participants sensed uncertainties of the situation would lead to challenges in safe/institutional delivery and feared deprivation of maternal benefits provided at the public

511 health services. Interestingly, most respondents thought of institutional delivery in terms of the benefits it offered (i.e., gain framing) and not in terms of the adverse outcomes it could help avoid (i.e., risk or loss aversion framing). Reduced interactions with the FLHWs left women without any support for planning childbirth, including options of the hospitals and the means of transport. Having limited resources further made it uncertain for women to access private health care. We observed that due to an absence of a clear directive or the regular schedule of contacts with health workers, there was a pessimistic outlook and a lack of initiative among pregnant women to take steps towards planning for delivery. This indicated an all-or-nothing, or absolutist mindset, a common cognitive distortion.

\section{Conclusion}

As discussed in this paper, the COVID-19 pandemic created distress and anxiety in the community, particularly among pregnant women whose plans for delivery were thrown into disarray owing to the lockdown and negligible interface with frontline health workers. However, the most overwhelming cause of anxiety was procuring adequate food for their families while their spouses' livelihoods suffered and not of contracting COVID-19. Since the pandemic was unprecedented, there was great uncertainty around when life would return to a state of normalcy. The pandemic

527 also brought to the fore a mistrust among end-users in the institutional healthcare system and its representatives due to the rapid spread of COVID-19 and high rates of fatality, unavailability of non- 

proliferation of half-information on preventing and managing COVID-19. To bridge the gap the

531 pandemic has created, there is a need for a refurbishment of the healthcare system with a focus on

532 arranging undisrupted ANC services with the dissemination of the correct information to the 533 grassroots and enabling FLHWs with more autonomy for decisions during their interactions.

534 This warrants for the continued service delivery and sustained trust in the system by the end-users.

535 The findings of this study will inform uptake of behavioural approach by stakeholders in 536 ensuring that ANC services are available and accessible in times of crisis. However, more 537 intervention-based evidence is needed to provide a comprehensive understanding of barriers to 538 ANC service provision to inform behavioural strategies to better address the problem.

\section{DECLARATIONS}

- Ethics approval and consent to participate Not Applicable corresponding author on reasonable request. 
The authors declare that they have no competing interests.

553

554

555

556

557

558

559

560

561

562

563

564

565

566

567

568

\section{- Funding}

This study was funded by the Bill and Melinda Gates Foundation as a part of the COVID19 work Centre for Social and Behaviour Change, Ashoka University was committed to. The funders had no role in study design, data collection, analysis and interpretation of data, or manuscript writing.

\section{- Authors' contributions}

SB and DG conducted the primary research, analysed and interpreted the qualitative data presented here. SB was a significant contributor in drawing the framework of the manuscript. All authors read and approved the final manuscript.

\section{- Acknowledgements}

The authors acknowledge the institutional support and resources provided by the Centre for Social and Behaviour Change, Ashoka University, in conducting this research and writing this paper. The authors would like to thank Dr Pavan Mamidi and Dr Sharon Barnhardt for reviewing the ideas presented here. The authors acknowledge the efforts of Aditya Laumas, Shreya Hasurkar, Nishtha Tewari, Parika Kumar and Pritha Sengupta in the data collection exercise conducted in Jharkhand, Madhya Pradesh and Uttar Pradesh. The authors acknowledge the effort of Parika Kumar in the literature review.

\section{- Authors' information}




\section{References}

1. WHO. Recommendations on Antenatal Care for a Positive Pregnancy Experience. [Internet]. WHO; 2016 [cited 2021 Oct 10]. Available from: https://www.who.int/publications/i/item/9789241549912

2. Sample Registration System Office of Registrar General of India. Special Bulletin on maternal mortality in India 2014-2016 [Internet]. India: Sample Registration System Office of Registrar General of India; 2018 [cited 2021 Oct 11] p. 1-3. (Maternal Mortality). Available from:

https://censusindia.gov.in/vital_statistics/SRS_Bulletins/MMR\%20Bulletin-201416.pdf

3. Ministry of Health and Family Welfare Government of India. National Rural Health Mission - Meeting people's health needs in rural areas. Framework for Implementation [Internet]. 3. Ministry of Health and Family Welfare Government of India.; 2005 [cited 2021 Dec 10]. Available from: http://nhm.gov.in/WriteReadData/1892s/nrhm-framework-latest.pdf

4. Ministry of Health and Family Welfare Government of India. National Urban Health Mission - Framework for Implementation. [Internet]. Ministry of Health and Family Welfare Government of India; 2013. Available from: http://www.nhm.gov.in/images/pdf/NUHM/Implementation_Framework_NUHM.pdf 5. Ministry of Health and Family Welfare Government of India. A strategic approach to reproductive, maternal, newborn, child and adolescent health $(\mathrm{RMNCH}+\mathrm{A})$ in India. 
[Internet: Ministry of Health and Family Welfare Government of India; 2013 [cited

599

600

601

602

603

604

605

606

607

608

609

610

611

612

613

614

615

616

617

618

619

620

621 2021 Dec 12]. Available from:

http://nhm.gov.in/index 1.php?lang=1\&level=1\&sublinkid=794\&lid=168

6. Tripathy P, Nair N, Sinha R, Rath S, Gope RK, Rath S, et al. Effect of participatory women's groups facilitated by Accredited Social Health Activists on birth outcomes in rural eastern India: a cluster-randomised controlled trial. Lancet Glob Health. 2016 Feb;4(2):e119-28.

7. Lyngdoh T, Neogi SB, Ahmad D, Soundararajan S, Mavalankar D. Intensity of contact with frontline workers and its influence on maternal and newborn health behaviors: cross-sectional survey in rural Uttar Pradesh, India. J Health Popul Nutr. 2018 Dec;37(1):2.

8. Seth A, Tomar S, Singh K, Chandurkar D, Chakraverty A, Dey A, et al. Differential effects of community health worker visits across social and economic groups in Uttar Pradesh, India: a link between social inequities and health disparities. Int J Equity Health. 2017 Dec;16(1):46.

9. Ministry of Health and Family Welfare,PIB Delhi. State/ UT Wise Details of Maternal Mortality Ratio (MMR) During Last Three Years Period. Press release [Internet]. 2021 Dec 2 [cited 2021 Dec 12]; Available from: https://www.pib.gov.in/PressReleasePage.aspx?PRID=1697441

10. Ministry of health and family welfare. Government of India. India fact sheet National Family Health Survey (NFHS-4) [Internet]. India: IIPS; 20152016 [cited 2021 Dec 12]. (NFHS). Available from: http://rchiips.org/nfhs/factsheet_nfhs-4.shtml 11. Ministry of Health and Family Welfare, Government of India. India fact sheet National Family Health Survey (NFHS-5) [Internet]. New Delhi: IIPS; 20192020 
[cited 2021 Dec 14]. (NFHS). Available from: http://rchiips.org/NFHS/NFHS5_FCTS/NFHS-5\%20State $\% 20$ Factsheet $\% 20$ Compendium_Phase-I.pdf

12. ICMR - National Institute for Research in Reproductive Health. Guidance for Management of Pregnant Women in COVID-19 Pandemic [Internet]. ICMR National Institute for Research in Reproductive Health; 2020 [cited 2021 Dec 14]. Available from: https://www.icmr.gov.in/pdf/covid/techdoc/Guidance_for_Management_of_Pregnant _Women_in_COVID19_Pandemic_12042020.pdf

13. Green L, Fateen D, Gupta D, McHale T, Nelson T, Mishori R. Providing women's health care during COVID-19: Personal and professional challenges faced by health workers. Int J Gynecol Obstet. 2020 Oct;151(1):3-6.

14. Ramila Bisht, Jyotishmita Sarma, Rajashree Saharia. COVID-19 lockdown: Guidelines are not enough to ensure pregnant women receive care. The Wire [Internet]. 2020 May [cited 2020 Dec 14]; Available from: https://thewire.in/women/covid-19-lockdown-pregnant-women-childbirth 15. Shrivastava S, M. S. Obstetric violence during COVID-19 is yet another challenge for Indian women. The Wire [Internet]. 2020 Apr; Available from: https://thewire.in/rights/women-covid-19-obstetric-violence

16. Aggarwal R, Sharma AK, Guleria K. Antenatal care during the pandemic in India: the problem and the solutions. Int J Pregnancy Child Birth. 2021 Feb 22;7(1):15-7.

17. Renuka Motihar. The impact of COVID-19 on reproductive health services. India Development Review [Internet]. 2020 Jun [cited 2021 Dec 12];(Online). Available from: https://idronline.org/the-impact-of-covid-19-on-reproductive-health-services/

18. Nguyen PH, Kachwaha S, Pant A, Tran LM, Walia M, Ghosh S, et al. COVID-19 Disrupted Provision and Utilization of Health and Nutrition Services in Uttar Pradesh, 
India: Insights from Service Providers, Household Phone Surveys, and Administrative Data. J Nutr. 2021 Aug 7;151(8):2305-16.

649

650

651

652

653

654

655

656

657

658

659

660

661

662

663
19. Sukumaran SAB, Manju L, Vijith D, Jose R, Narendran M, John S, et al. Protective Behaviour against COVID 19 and Telemedicine Use among the Pregnant Women during Pandemic Period: A Cross Sectional Study. Int J Trop Dis Health. 2020;44-52.

20. Barbosa-Leiker C, Smith CL, Crespi EJ, Brooks O, Burduli E, Ranjo S, et al. Stressors, coping, and resources needed during the COVID-19 pandemic in a sample of perinatal women. BMC Pregnancy Childbirth. 2021 Dec;21(1):171.

21. Mortazavi F, Ghardashi F. The lived experiences of pregnant women during COVID19 pandemic: a descriptive phenomenological study. BMC Pregnancy Childbirth. 2021 Dec;21(1):193.

22. Tong A, Sainsbury P, Craig J. Consolidated criteria for reporting qualitative research (COREQ): a 32-item checklist for interviews and focus groups. Int J Qual Health Care. 2007 Sep 16;19(6):349-57. 